\title{
Interactive Kinect Designed for Mobile Phone into Education
}

\author{
Mahdieh Molaei Tehrani, Yan Peng Lim, and Ahmadreza Samavatian
}

\begin{abstract}
It is widely known that the interactive Kinect is defined as natural interaction device. The efficiency of technical interactivity can support the pedagogical practices to benefit learner to enhance classroom activity. Beside that personal mobile phones are now ubiquitous amongst student populations in universities. Students like mobile technology and use it regularly in their personal lives. It is no surprise that young people want to employ mobile devices to make education more engaging and particular needs. While the learning approach of students will strongly influence the quality of their learning outcomes. This project finds a path in order to develop a prototype to control Kinect through a mobile phone and interact with a computer on educational way. This can be considered as a useful tool educational environment.
\end{abstract}

Index Terms - Interactive, kinect, mobile phone, education, kinect, etc.

\section{INTRODUCTION}

Highlight the evolution of technologies to capture users interactions, oftentimes ubiquitously, created new paradigms to the interactive applications of interfaces conception. The Kinect is one of the most important commercial icons that characterize this new way of thinking about interfaces, making the user-machine interaction more natural.

It is not very convenient and flexible for users controlling the Kinect through computers or the Microsoft Xbox console. Although Microsoft has attempted to make interaction with the Kinect as convenient as possible, users still experience somedifficulty stemming mainly from the inaccuracy of the Kinect device, especially at reading inputdata. As Leyvand, Meekhof, Wei, Sun and Guo (2011) note, the biggest challenge of the Kinect device is inaccuracy. Any inaccuracy in reading input data results in inaccuracy in interpretation of data (i.e., visual representation of user activity). Nevertheless, as companies continued to realize the advantages of motion tracking they started to invest greater effort into research and development. An example of these companies is the Microsoft Company, which introduced the Kinect device in 2010. Although, the device was originally developed for the purpose of gaming, it is now known to be useful for a wide variety of purposes [1]. In recent years and by increase the demands on online markets for delivering knowledge-based products through network, the number of vendors who provide online educational products are increasing as well. There are also other products that can be used for education

Manuscript received June 6, 2014; revised August 12, 2014.

Mahdieh Molaei Tehrani and Yan Peng Lim are with the Multimedia University, Malaysia (e-mail: mahdieh.molaei@gmail.com, orestesther@gmail.com).

Ahmadreza Samavatian is with the Mahmood Abad University, the Islamic Republic of Iran (e-mail: ahmadreza.samavatian@gmail.com). although they are not technically designed for this matter. One of the main successfully commercialized products which can be occupied for different matters is Microsoft Kinect. It is not very convenient and flexible for users controlling the Kinect through computers or the Microsoft Xbox console.

\section{INTERACTIVE FOR KINECT}

Microsoft's Kinect sensor was originally developed as an accessory for the Xbox 360 videogame console that would allow interaction without touching a game controller, through natural postures and gestures (Microsoft Kinect for Xbox 360, 2012). Soon enough the scientific community realized that it could be applied to a wide range of applications. To test such applications is often a hard and time consuming activity because it is difficult to redo tests using the same reference input, and furthermore it requires considerable physical effort from the users to perform movements and body gestures.

\section{The KineCt DeVICE: ExPloRed AREAS APPLICATION}

The Kinect has already been tested in certain areas and applications. Oliver, Kang, Wünsche and MacDonald (2012) investigated the suitability of the Xbox Kinect optical sensor for navigation, simultaneous localization and mapping purposes. They noted that the limits to the range of applications in practice are due to the smaller field of view, depth range and higher processing requirements for the resulting sensor data. Both localization and mapping are essential requirements in mobile robotics to accomplish navigation. In most cases, laser scanners are used but they are expensive and are limited to 2D mapping capabilities.Another area where the Kinect has been useful is in Virtual Conferencing. Since the device is equipped with components that give it the ability to capture videos of a person, identify that person's body, and track the movements of their body in 3D space, research on using Motion Controllers in Virtual Conferencing was conducted. It sought to explore how 3D cameras with the capability to recognize and track movements of humans could be used to enhance meetings in a virtual world. The project came about in response to the release of the Kinect by Microsoft.

Also, there are many solutions available for interacting with the Kinect device using a computer.

\section{INTERACTIVE KINECT IN EDUCATION}

By now the most successful approach is to attract pre-university youth and university freshmen into those fields before they make a decision on future subjects by introducing 
them with interactive, modifiable and inspiring virtual environments. Beside that the location and workspace for motion capture is fixed. Besides, if interactions with not only haptic devices but also virtual avatars are needed, it will be necessary to have a more sophisticated environment incorporated with Human-Computer Interaction abilities [2].

Become a true innovator in the classroom by introducing a new style of teaching that interacts, informs and engages your students. Microsoft's Kinect motion-sensor device, which works with an Xbox 360 gaming console, offers nearly limitless opportunities to introduce game-based learning to let your students' creativity soar. You can use existing Kinect in education in creative ways, or use the free Kinect for Windows Software Development Kit (SDK) to create custom learning opportunities for your students. Either way, Kinect offers an exciting new tool that can help you transform ordinary classroom activities into extraordinary learning experiences.

In addition, the Kinect for Windows SDK lets technically-inclined teachers and emerging computer science students create customized applications that further develop technical knowledge.

\section{Mobile FunCTIONALITY AND USER INTERACTION}

The last few years have seen a tremendous increase and change in mobile computing, and average mobile consumption rates are extremely high. The number of people who rely on their mobile phones for various services is increasing rapidly as the devices become more sophisticated and afford multiple uses. New mobile devices provide users with much different functionality that makes basic life activities convenient, fun and efficient.

Mobile functionality refers to the features and applications that help users perform activities and operations in a manner that meets their objectives. Various studies such as by Rodden, Cheverst, Davies and Dix (1998), Hinckley, Pierce, Sinclair and Horvitz (2000), Dunlop and Brewster (2002) and Luket al., (2006) explore the usability of mobile devices for different purposes. Roddenet al., stated that interaction is not merely an attribute of the mobile device but instead it is strongly dependent on the context in which the device is being used [3]-[5].

\section{INTERACTIVE KINECT USING MOBILE PHONE IN EDUCATION}

The Kinect has proven to be simple to use and understand; however, it still has some problems, asits functionality is not yet fully developed. In a related studyattempted to explore the concept of Smartphone sensors enhancing the Kinect experience and noted some problems with the Kinect. More specifically, they identified the following major issues:

The result from the study shows positively that the particular part of the body measured by the Kinect is dependent on the way in which the smartphone is held by the user. Nevertheless, the study is limited in that the degree to which the Kinect can be supported by the mobile is not determined [6].

Due to the fact this is a completely new technology and paradigm of interaction and the researcher had to cope with some problems initially that didn't expected. The fact this is a fresh technology and the entire world directly connected is also fresh itself could initially create some problems and it can be a little bit destabilized, this research the possibility to build concrete tools is already a reality. Certainly this technologyneeds to be improved a lot before becoming a stable tool in the educational world but it is in rapid development, hence early that will see the first Kinect-based applications appearing in education.

In addressing the problem of occlusion, Dockstader and Tekalp (2001) introduced a near real-time solution by using a probabilistic mixing of low level features and components. As described by the researchers, "the algorithm mixes coarse motion estimates, change detection information, and unobservable predictions to create more accurate trajectories of moving objects." The findings from their experiment show that using the method reduces the inaccuracy of the device.

\section{AFFORDANCE OF BENEFIT OF KINECT IN CLASSROOM}

In order to have a Kinect in the classroom the researcher need to have a Kinect device, a video projector and proper software specifically for our purpose. The first two can be afforded easily from the market but about the software there should be mentioned that there is only limited number of applications developed for this manner. One of the best developed systems is from Evoluce company in Germany by the name of Win\&Iapplication which gives user access to control desktopapplications and some of Microsoft software products by recognizing his/her gestures within windows 7 [7].

With all respect to their attempts but there is not much applications to use Kinect's potential to be used in for education.

The cost of Kinect in is very low in compare of its competitors that we made a comparison of cost:

There are two differences make it to be preferred by developers:

1) Kinect is using image-processing instead of motion sensor data processing and it can make user feel comfortable and free because he/she shouldn't carry the device with himself/herself to keep interacting.

2) Kinect's engine works with $\mathrm{C}++$ language which enables application developers to write or modify software if they are interested. This is not possible in other systems.

3) Kinect has an SDK (Software Development Kit) that let the programs access to all hardware features that the other two don't [8].

\section{TESTING Procedures IN EdUCATION}

The main experiment was comprised of sessions with 10 students of both genders, aged range from 8-12 and mainly 18-30 years old, recruited from students of Faculty of Creative Multimedia University. After the orientation and pre-questionnaire sessions, students wereasking to test the application.

At the start of the sessions, students was given a chance how to use the windows mobile phone and navigate to move 
the Kinect sensor.

They were then trained to spend their time to work and interacting with kinect through mobile phone.

The main experiment was designed to test the effect of different interactivity features, in terms of student's subjective ratings of their Interest. The effect of interactivity features, in terms of users'collaborative interaction; and the effect of individual differences (e.g. gender). The process starts by building a multicast group and join nodes once there is a need. In case a send message procedure needed, the node has to connect and join the group which is created before and send the message within the group [9]-[11].

It is obvious that all nodes will be able to receive it. The main advantage of this for this project purpose is a system which is able to operate even if there was no internet connection and through a local network only.The group of students in class can study as collaborative and have discussion as a group when they use their mobile phone as a tool to interact with Kinect.

As shown in Fig. 1 bellow, this application may use in all schools and university to teach all subject with interactive Kinect through mobile phone.

The group of students in class can study as collaborative and have discussion as a group when they use their mobile phone as a tool to interact with Kinect. This application may use in all schools and university to teach all subject with interactive Kinect through mobile phone and can be any file format.

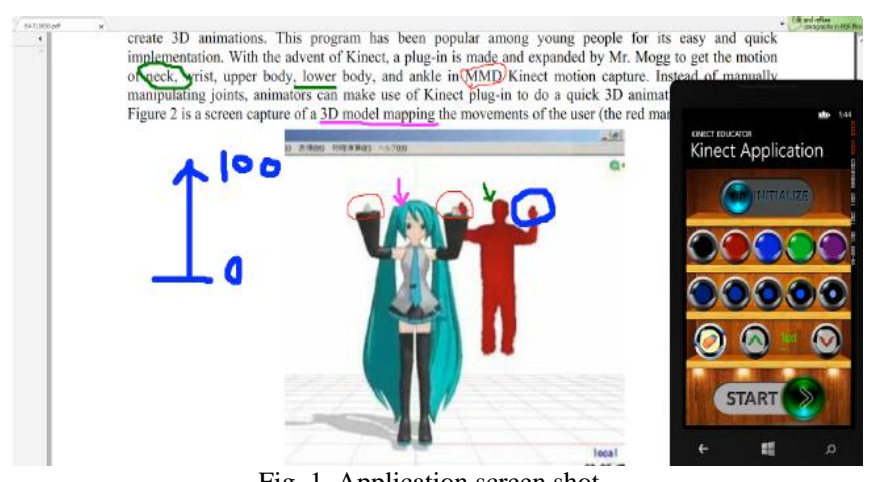

Fig. 1. Application screen shot.

In a more general view we can say that there are three layers of control, interface and network are handling the entire process of a user interaction with the designed system.

This application has initialize button in order to initialize Kinect device and make the device ready to recognize user and extract the body skeleton in order to track the hand point position.

Fig. 2 shows button's shapes and colors change on pressing each one of them in order to show user which one is selected. It is obvious that in each category only one of the buttons can be selected. In first row after pressing the "Initialize" button the color and text of button changes so user knew that the device is ready to capture and track. The change of button which shown on Fig. 2.

This application has initialize button in order to initialize Kinect device and make the device ready to recognize user and extract the body skeleton in order to track the hand point position.

Button's shapes and colours change on pressing each one of them in order to show user which one is selected. It is obvious that in each category only one of the buttons can be selected. In first row after pressing the "Initialize" button the colour and text of button changes so user knew that the device is ready to capture and track. The change of button has shown below.

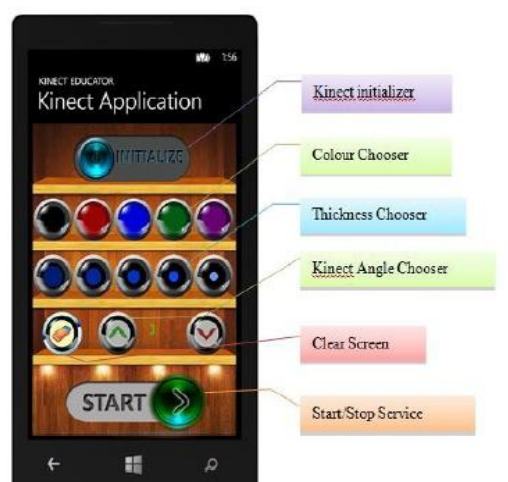

Fig. 2. Windows phone application components.

The same change goes to the rest of buttons. In second row if there is a color selected the button will have a yellow ring around. It is the same for the third row as well but for choosing the thickness of the pen.

\section{DATA ANALYSIS}

The type of students' interactions and the degree of such interaction depends on the decision of their teacher and the kind of study material provided for them. As comes from Table I most of students believe that this type of interaction had increased their participation in discussions and collaboration inside the classroom.

TABLE I: INTERACTIVITY AND PARTICIPATION IN LESSON

\begin{tabular}{|ccccccc|}
\hline \multicolumn{7}{|c|}{ Interactivity and participation in lesson by total } \\
\hline Points & $\mathbf{1 8 - 2 0}$ & $\mathbf{2 1 - 2 3}$ & $\mathbf{2 4 - 3 0}$ & Below $\mathbf{1 8}$ & 0ver $\mathbf{3 0}$ & Total \\
\hline $\mathbf{1}$ & $\mathbf{0 . 2 6 \%}$ & $\mathbf{0 . 0 0 \%}$ & $\mathbf{0 . 0 0 \%}$ & $0.00 \%$ & $0.00 \%$ & $0.26 \%$ \\
\hline $\mathbf{2}$ & $2.10 \%$ & $1.57 \%$ & $1.57 \%$ & $0.52 \%$ & $0.00 \%$ & $5.77 \%$ \\
\hline $\mathbf{3}$ & $15.75 \%$ & $2.36 \%$ & $2.36 \%$ & $0.00 \%$ & $2.36 \%$ & $22.83 \%$ \\
\hline $\mathbf{4}$ & $15.75 \%$ & $4.20 \%$ & $3.15 \%$ & $1.05 \%$ & $1.05 \%$ & $25.20 \%$ \\
\hline $\mathbf{5}$ & $27.56 \%$ & $7.87 \%$ & $3.94 \%$ & $2.62 \%$ & $3.94 \%$ & $45.93 \%$ \\
\hline Total & $\mathbf{6 1 . 4 2} \%$ & $16.01 \%$ & $11.02 \%$ & $\mathbf{4 . 2 0} \%$ & $\mathbf{7 . 3 5} \%$ & $100.00 \%$ \\
\hline
\end{tabular}

In order to summarize the data analysis researchers, have collected to come to a proper conclusion. We put a data filter on all the results came from students. The filter limits the results to those who have ranked the system with numbers of " 3 " or " 4 " or " 5 ". The result shows that more than half of participant of video recording interview, are agree with the pedagogical factors we have discussed in the video recording interview. This means the result certifies even with the current condition of design and multimedia, interactivity, efficiency that let students can accept it to exist in classroom.

\section{CONClusion}

The aim of this research was to understand the possibility to introduce the interactive Kinect as a concrete tool for mobile phone to support teaching activities in classroom. The obtained results are satisfactory and they enable us to say we were able to achieve the objectives that fixed in advance 
before starting to develop this project.

Due to the fact this is a completely new technology and paradigm of interaction and the researcher had to cope with some problems initially that didn't expected. The fact this is a fresh technology and the entire world directly connected is also fresh itself could initially create some problems and it can be a little bit destabilized, this project the possibility to build concrete tools is already a reality. Certainly this technologyneeds to be improved a lot before becoming a stable tool in the educational world but it is in rapid development, hence early that will see the first Kinect-based applications appearing in education.

This project is the first step in the complex process to create Kinect-based application, using mobile phone in education.

Certainly in the future the hardware and the frameworks designed to interact with it will be subjected to huge improvements. Hence it can leave to be one of possible directions that can take to continue exploring this amazing field of research. The future work of this project can be multiple users and can use in any kind of device.

\section{REFERENCES}

[1] T. Leyvand, C. Meekhof, Y. C. Wei, J. Sun, and B. Guo, "Kinect identity: Technology and experience," Computer, vol. 44, no. 4, pp. 94962011.

[2] L.Wei, H. Zhou, A. K. Soe, and S. Nahavandi, "Integrating Kinect and haptics for interactive STEM education in local and distributed environments," in Proc. 2013 IEEE/ASME International Conference on Advanced Intelligent Mechatronics (AIM), 2013, pp. 1058-1065.

[3] C. Y. Lin, M. Wu, J. A. Bloom, I. J. Cox, and M. Miller, "Rotation, scale, and translation resilient public watermarking for images," IEEE Trans. Image Process, vol. 10, no. 5, pp. 767-782, May 2001.

[4] T. Rodden, K. Cheverst, K. Davies, and A. Dix, "Exploiting context in HCI design for mobile systems," Workshop on Human Computer Interaction with Mobile Devices, 1998, pp. 21-22.

[5] K. Hinckley and D. Wigdor, "Input technologies and techniques," The Human-Computer Interaction Handbook: Fundamentals, Evolving Technologies and Emerging Applications, 2002, pp. 151-168.
[6] M. Dunlop and S. Brewster, "The challenge of mobile devices for human computer interaction," Personal and Ubiquitous Computing, vol. 6, no. 4, pp. 235-236, 2002.

[7] M. H. Haeusler, "Unpacking the full potential of media facades through a fusion with Kinect technology," International Journal of Knowledge-Based Development, vol. 4, no. 2, pp. 141-156, 2013.

[8] J. Kim, J. Park et al., "HCI (human computer interaction) using multi-touch tabletop display," in Proc. IEEE Pacific Rim Conference on Communications, Computers and Signal Processing, 2007, pp. 391-394.

[9] W. Kung-Teck and R. Osman, "Affordances of interactive whiteboards and associated pedagogical practices: Perspectives of teachers of science with children aged five to six years," Turkish Online Journal of Educational Technology, vol. 12, no. 1, pp. 1-8, 2013.

[10] P. Robinson, A. Mackey et al., "Attention and awareness in second," in The Routledge Handbook of Second Language Acquisition, S. Gass and A. Mackey, Eds., New York: Routledge, 2013, pp. 247-267.

[11] R. S. Pressman, Software Engineering (A Practitioner's Approach), McGraw-Hill Higher Education, 2009.

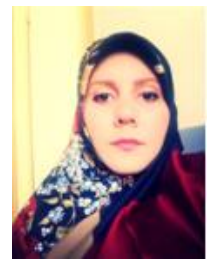

Mahdieh Molaei Tehrani was born in Tehran in 1982 She was graduated from Multimedia University Malaysia in master of e-learning. She received her master of multimedia e-learning technologies, from the Faculty of Creative Multimedia, Multimedia University, (2011-2013); and the bachelor of digital media, the Faculty of Creative Multimedia, Multimedia University, (2003-2007). She has published several papers in international journals

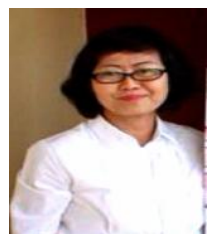

Yan Peng Lim was born in Malaysia in 1963. She was graduated from California College of Arts in 1992 in field of graphic design and she got her master of fine arts from Yale University USA in 1995 in field of graphic design. She got her doctor of philosophy in 2010 from Multimedia University Malaysia. She has published several papers in international journals. Her research interest include digital media and virtual reality.

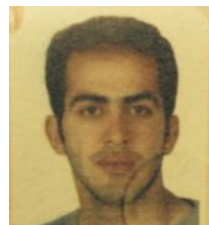

Ahmadreza Samavatian was born in 1987 in Tehran He was graduated from the Mahmood Abad University from Islamic Republic of Iran in 2011 in field of information technology. He is currently studying his master of computer science at Islamic Azad University. $\mathrm{He}$ is interested in information technology and computer science. 